\title{
Caractérisation hydro chimique des eaux souterraines de la region de Tahoua (Niger)
}

\author{
Haoua Amadou, Mahaman Sani Laouali, Abdou Salam Manzola \\ Laboratoire de Chimie de l'eau, Faculté des Sciences et Techniques \\ Université Abdou Moumouni de Niamey, Bp 10662 Niamey-NIGER \\ Auteur correspondant e-mail : haoua_amadou2000@yahoo.fr
}

Original submitted in on 24t June 2014. Published online at www.m.elewa.org on $30^{\text {th }}$ September 2014. http://dx.doi.org/10.4314/jab.v81i1.6

\section{RESUME}

Objectif: L'étude s'intéresse à la qualité des eaux de cinq aquifères (les nappes alluviales, le continental Terminal, le continental Intercalaire, le crétacé marin et les formations marines) de la région de Tahoua. Méthodologie et résultats : Une série d'analyses physico-chimiques et microbiologiques a été réalisée sur les ressources en eaux de la zone. Les résultats de ces analyses ont été traités à partir d'une méthode hydro chimique qui utilise le diagramme triangulaire de Piper et des méthodes statistiques multi variées dont l'Analyse en Composantes Principales Normées (ACPN) et la Classification Hiérarchique Ascendante (CHA). Le Diagramme triangulaire de Piper montre que les eaux de la zone d'étude se répartissent en quatre principales familles. II s'agit des eaux bicarbonatées calciques et magnésiennes, plus importantes et représentant $60,87 \%$, les eaux sulfatées calciques et magnésiennes $21,74 \%$, les eaux chlorurées calciques et magnésiennes 13,04\%, et les eaux sulfatées sodiques 4,36\%. L'Analyse en Composantes Principales Normées et la Classification Ascendante Hiérarchique indiquent que la minéralisation des eaux de la zone est contrôlée par trois grands phénomènes : la minéralisation-temps de séjour qui se traduit par l'hydrolyse des minéraux silicatés pour les eaux souterraines, le pluviolessivage des sols et l'influence des activités humaines dans la production des déchets polluants. Cette étude montre également que les ressources en eau étudiées de la zone sont fortement influencées par les activités anthropiques, avec la présence des coliformes fécaux de type Escherichia coli et de streptocoques fécaux qui sont la conséquence d'une pollution d'origine humaine récente.

Conclusion et application des résultats : Cette étude révèle que les eaux souterraines de la région de Tahoua subissent une pollution anthropique importante. Dans son ensemble, ces eaux souterraines nécessitent dans certains cas, un traitement spécifique avant approvisionnement.

Mots clés : Analyse en composantes principales, Classification hiérarchique Ascendante, Diagramme de Piper, Hydrochimie et eaux souterraines.

\section{Hydro chemical characterization of groundwater in area of Tahoua (Niger) \\ Abstract}

Objective: This study investigated the quality of water of five aquifers (Alluvial aquifer, continental terminal aquifer, "continental Intercalaire" aquifer, Cretaceous seaway and Marine aquifer formation) of the region of Tahoua. 
Methodology and results: A set of physico-chemical and microbiological analyses were done at the water resources of the zone. The results of these analyses were treated by a hydro chemical method that uses the Piper triangle diagram and multivariate statistical methods including Principal Component Analysis normalized (PCA) and Hierarchical Ascending Classification (HAC).The Piper triangular diagram showed that the waters of the study area are divided into four main families. The most important calcium and magnesium bicarbonate waters, representing $60.87 \%$, calcium and magnesium sulfate waters $21.74 \%$, the calcium and magnesium Chloride $13.04 \%$, and sodium sulfate waters $4.36 \%$. The Principal Component Analysis standardized and Agglomerative Hierarchical indicate that mineralization of the waters of the area is controlled by three major factors: the mineralization-residence time which results in the hydrolysis of silicate minerals in the groundwater, soil leaching and the influence of human activities in the production of polluting waste. This study also shows that the water resources of the area are strongly influenced by human activities, with the presence of fecal coliform Escherichia coli and fecal streptococci that are the result of recent anthropogenic pollution.

Conclusion and application of results: This study reveals that the groundwater resources of the region of Tahoua are subject to high anthropogenic pollution and need in certain cases a specific treatment before supply.

Keywords: Principal component analysis, Ascending Hierarchical clustering, Graph Piper Hydrochemistry and groundwater.

\section{INTRODUCTION}

L'eau souterraine est d'une importance capitale dans la plupart des régions du monde. Toutefois, cette ressource qui était jadis de bonne qualité, se trouve actuellement menacée par diverses sources ponctuelles et diffuses de contamination. Les eaux souterraines constituent la principale source d'approvisionnement des habitants de la région de Tahoua (Maoudé, 1988). Depuis une vingtaine d'années, cette région connaît un important essor économique avec un développement de l'activité agricole et surtout de l'élevage, associé à une forte expansion démographique. L'eau qui constitue un élément incontournable pour le développement économique est menacée de contamination. II convient donc de connaître et suivre la qualité de cette ressource (Boubakar, 2010). La composition chimique d'une eau issue du milieu naturel est très variable. Elle dépend de la nature géologique du sol d'où elle provient et aussi des substances réactives qu'elle aurait pue rencontrer lors de l'écoulement. Ainsi la composition quantitative et qualitative de l'eau souterraine en matières en suspension et dissoutes, de nature minérale ou

\section{MATERIEL ET METHODES}

Présentation de la zone d'étude: La région de Tahoua (Niger) couvre une superficie de $113317 \mathrm{~km}^{2}$. organique, détermine sa qualité (Jain et al., 2005). Cependant cette qualité peut être altérée lorsque des substances extérieures entrent en contact avec la nappe aquifère. Tel est le cas des substances indésirables voire toxiques qui rendent l'eau souterraine impropre et toxique pour divers usages notamment pour l'usage comme eau de boisson. L'utilisation intensive des ressources naturelles et l'accroissement des activités humaines ont engendré de graves problèmes sur la qualité des eaux souterraines (Foster, 1995 ; Mor et al., 1995). L'étude hydro chimique des eaux a pour but d'identifier les faciès chimiques des eaux, leur degré de potabilité, ainsi que leur aptitude à l'irrigation. Elle permet de suivre aussi l'évolution spatiale des paramètres physicochimiques et d'estimer leur origine minérale. Des études hydrogéologique et physico-chimique des eaux souterraines seront intégrées et employées pour déterminer d'une part l'influence de ces facteurs et de l'autre les mécanismes contrôlant la chimie des eaux souterraines dans la région.

La région dont la latitude Nord est comprise entre $13^{\circ}$ $40^{\prime}$ et $19^{\circ}$, et la longitude Est entre $4^{\circ}$ et $12^{\circ} 40^{\prime}$ est 
entièrement en zone climatique sahélo-soudanienne, ayant pour limites extrêmes des précipitations, $150 \mathrm{~mm}$ et $600 \mathrm{~mm}$. Des températures varient de $10^{\circ}$ (harmatan) à $45^{\circ}$ (saison chaude).

Hydrogéologie: L'hydrogéologie de Tahoua est complexe. Nous avons une superposition d'une multitude de nappes dans la bande Sud. Leurs débits varient d'une zone à une autre. C'est ainsi qu'on peut avoir des ouvrages très productifs $\left(20 \mathrm{~m}^{3} / \mathrm{h}\right)$ à des faibles profondeurs, comme on peut avoir des débits aléatoires dans la même nappe mais avec une implantation très décalé. Cela est dû au pendage et au mode de formation géologique de la nappe. Les principales nappes sont :

Les nappes alluviales: Ce sont des dépôts sédimentaires (limons, sable, gravier, condomérat) qui proviennent de la dégradation ou du remaniement d'autres roches qui se déposent au fil du temps sur un terrain imperméable et encaissé.

Ils sont déposés dans ces encaissements et forment le lit des vallées. Les principales vallées de la Région sont : la Tarka, Badaguichiri, Maggia, Bagga et Tadis.

Les nappes du continental Terminal (CT1): Elles affleurent sur la bordure à l'est de la ligne Birni N'KoniTahoua. Sur la bordure occidentale, le Continental Terminal repose en discordance sur les altérites du socle antécambrien alors qu'au sud elle repose sur le Continental Hamadien (Greigert, 1966). Les nappes du CT1 sont très importantes et ont un débit très élevé. Ces nappes sont aussi alimentées par l'eau de pluie. Le CT couvre une grande superficie (Département d'lllela, de Birni N'konni, de Bouza, de Keita, de Tahoua et de Madaoua). Mais ces nappes sont plus productives dans Illela, Birni N'konni et le Sud du département de Tahoua. Le dépôt se compose des grés ferrugineux, de kaolinite, du sable et des marres.

Le continental Intercalaire/Hamadien $(\mathrm{CIH})$ : La nappe $\mathrm{du} \mathrm{Cl} / \mathrm{H}$ est la plus grande, la plus importante en débit et en étendue. Elle couvre toute la Région de Tahoua. Elle est captée parfois à plus de $8010 \mathrm{~m}$ au Sud-Ouest par des forages profonds. Avec un niveau statique compris entre 60 à $100 \mathrm{~m}$. Dans le département d'Abalak le Cl est captable par les puits de $125 \mathrm{~m}$. C'est une nappe captive au Sud mais libre au Nord où la formation affleure.

Les nappes du crétacé: Elles sont formées de nappes localisées plus ou moins fossiles. Elles se retrouvent dans la totalité de la région à plus de $400 \mathrm{~m}$ généralement vers le Sud-Ouest mais sont superficielles vers le Nord à $80 \mathrm{~m}$ en moyenne. Les dépôts sont composés de calcaire, d'argile, de silt et de sable.

Méthode expérimentale: Les analyses physicochimiques ont été réalisées sur les eaux de forages et puits captant les nappes des Alluviales (fond de Kori, bas fond et Maggia), du Continental Intercalaire, du Continental Terminal, du Crétacé marin et des Formations marines de la région de Tahoua. Les prélèvements sont réalisés et conditionnés dans des flacons spécialement préparés à cet effet. Les prélèvements pour les forages munis de robinets sont effectués après avoir fait couler l'eau dans le vide pendant environ trois minutes. Les flacons d'échantillonnage sont ensuite remplis à des fins d'analyse. Pour les puits, les prélèvements sont effectués à l'aide d'un échantillonneur spécialement conçu. Le dispositif d'échantillonnage est soigneusement lavé à l'eau distillée avant chaque prélèvement. La quasi-totalité des points d'eau des aquifères est destinée à l'alimentation en eau potable et pour l'irrigation des terres agricoles. Pour être utilisée l'eau doit répondre à certaines normes qui varient en fonction du type d'utilisation. Les échantillons d'eau destinés aux analyses chimiques ont été prélevés dans vingt-trois (23) cantons de la région de Tahoua (Figure 1). Pour chaque test, les paramètres physiques à savoir le $\mathrm{pH}$, la température, la conductivité, la turbidité et la couleur, sont mesurés in situ à l'aide d'un pH-mètre et d'un conductimètre de marque WTW 330.Les échantillons d'eau ont été immédiatement stockés à $4^{\circ} \mathrm{C}$ dans une glacière contenant de la glace, l'analyse a été effectuée rapidement moins de $24 \mathrm{~h}$ après le prélèvement. Les éléments majeurs fer $\left(\mathrm{Fe}^{2+}\right)$, Sodium $\left(\mathrm{Na}^{+}\right)$, chlorure $(\mathrm{Cl}$ ), sulfate $\left(\mathrm{SO}_{4}{ }^{2-}\right)$, Fluorure $\left(\mathrm{F}^{-}\right)$; bicarbonate $\left(\mathrm{HCO}_{3}{ }^{-}\right)$, nitrate $\left(\mathrm{NO}_{3}{ }^{-}\right)$, nitrite $\left(\mathrm{NO}_{2}{ }^{-}\right)$, dureté sont analysés dans le chef-lieu de la région où un laboratoire a été spécialement aménagé. Ces analyses ont été réalisées à l'aide d'un spectrophotomètre en utilisant les méthodes classiques préconisées par les normes françaises AFNOR. En ce qui concerne la microbiologie, l'échantillonnage est réalisé dans des tubes à essai stérilisés par autoclavage à $121^{\circ} \mathrm{C}$ pendant 20 minutes. Les paramètres analysés sont les coliformes fécaux et les streptocoques fécaux, qui sont celles dont l'étude est recommandée par l'OMS pour le cas des eaux de boisson. La méthode utilisée pour isoler et déterminer ces bactéries, est la technique par filtration. C'est une méthode très sensible qui permet de déterminer les bactéries à des faibles concentrations. 


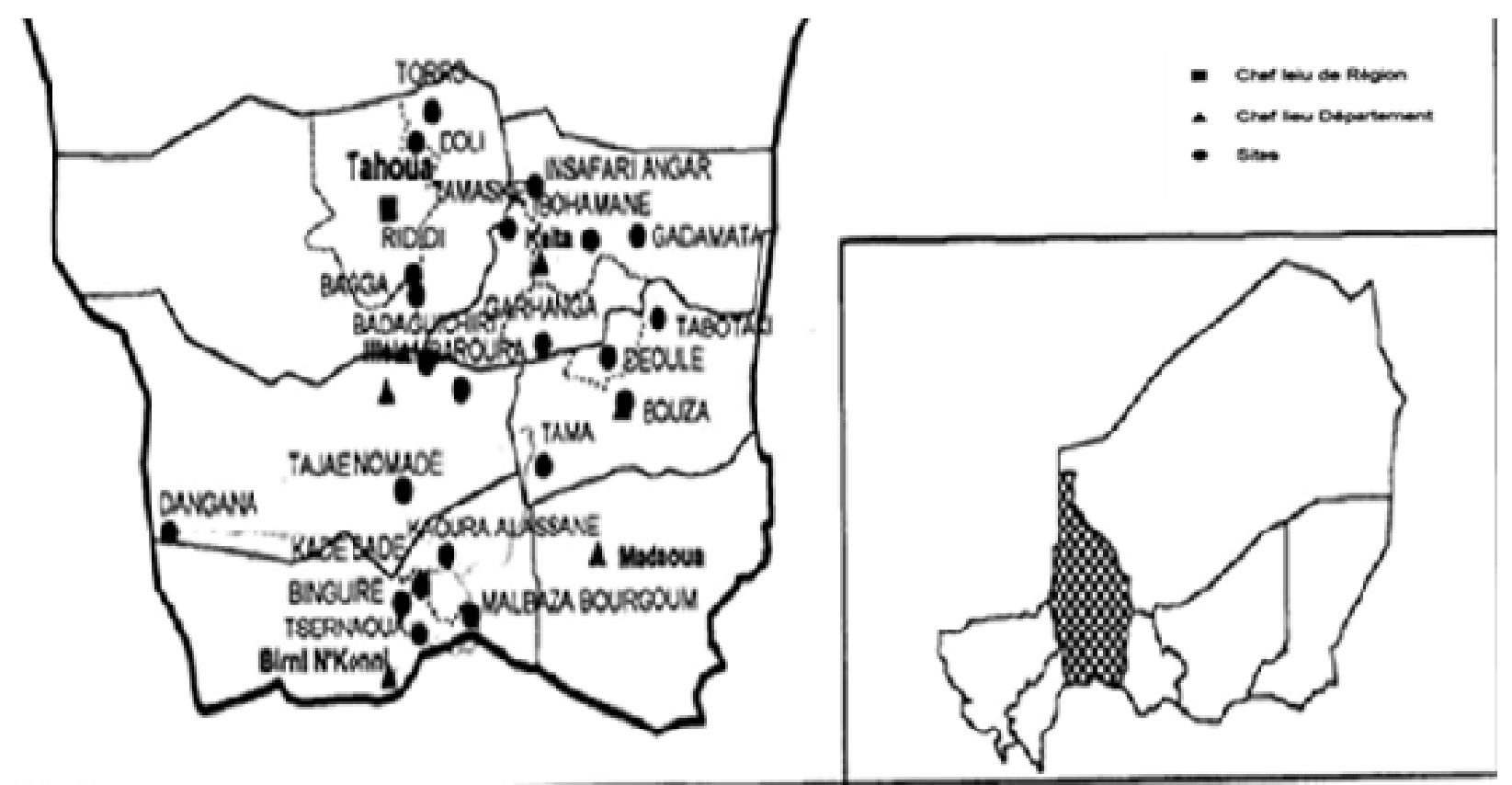

Figure 1 : Localisation des points de prélèvement.

Les aquifères échantillonnés se répartissent comme suit :

- $\quad$ Les Alluviales de fond de Kori, de Bas fond et de la Maggia avec six (6) puits échantillonnés dans les différentes localités à savoir Binguire, Doli, Insafari, Gada Mata, Tama, Malbaza Bourgoum;

Le continental Intercalaire avec cinq (5) forages à Keita, Tamaske, Bouza, Ibo Hamane, Kade Bade.

- $\quad$ Le Continental Terminal avec sept (7) puits échantillonnés à (Gar Hanga, Rididi, Dangona, Badagui Chiri, Bagga, Kaoura Alassane, Tser Naoua).

- $\quad$ Les Formations marines avec cinq (5) puits à Tabotaki, Deoule, Tajae Nomade, Amba Roura et un échantillon à Toro sur le Crétacé marin. La potabilité de l'eau est définie par des paramètres physiques, chimiques et biologiques, mais surtout en fonction de son usage. Une comparaison des teneurs en éléments physiques et chimiques des eaux des différents forages aux normes de l'Organisation Mondiale de la Santé a été effectuée suivant Lallahem (2002). L'analyse hydrochimique a été ensuite réalisée à l'aide du digramme de Piper notamment pour caractériser les faciès géochimiques des eaux de la zone de Tahoua. Ce diagramme est très fréquemment utilisé et donne de très bons résultats (Yermani et al., 2003; Alayat et
Lamouroux, 2007; Kouassi et al., 2010 ; Ahoussi et al., 2011; Ahoussi et al., 2012; Ahoussi et al., 2013; Eblin et al., 2014 ). Le traitement a été possible grâce au logiciel DIAGRAMME. La seconde approche est basée sur l'utilisation de la statistique multi variée à partir de l'Analyse en Composantes Principales Normées (ACPN) et Classification Hiérarchique Ascendante (CHA).L'ensemble des données collectées sur les eaux souterraines de la région a fait l'objet de cette étude statistique. L'analyse statistique a été réalisée sur 23 échantillons et 12 variables (la conductivité électrique (CE), le pH, la dureté, la turbidité, $\mathrm{Na}^{+}, \mathrm{F}^{-}, \mathrm{Fe}^{2+}, \mathrm{Cl}^{-}$, $\mathrm{HCO}_{3}^{-}, \mathrm{SO}_{4}{ }^{2-}, \mathrm{NO}_{3}{ }^{-}$et $\mathrm{NO}_{2}{ }^{-}$) à l'aide du logiciel XLSTAT 2014. Cette analyse permet de synthétiser et de classer un nombre important de données afin d'en extraire les principaux facteurs qui sont à l'origine de l'évolution simultanée des variables et de leur relation réciproque (Biémi, 1992). Elle permet de mettre en évidence les ressemblances et la position graphique que présenteraient deux ou plusieurs variables chimiques au cours de leur évolution. Les différentes méthodes utilisées dans cette étude permettront de connaître d'une part le mécanisme de minéralisation des eaux des sites étudiés et de l'autre les relations qui existent entre ces ressources en eau et les activités anthropiques de la zone d'étude. 
RESULTATS ET DISCUSSION

Évaluation de la potabilité des eaux : La température des eaux de la région varie entre 24,40 et $37,80^{\circ} \mathrm{C}$, avec une moyenne de $29,73 \pm 1,58{ }^{\circ} \mathrm{C}$. En ce qui concerne le pH des eaux, il varie entre 6,46 et 7,81 unités $\mathrm{pH}$, pour une moyenne de 7,23 $\pm 0,44$. La conductivité électrique varie entre 49 et $1156 \mu \mathrm{S} . \mathrm{cm}^{-1}$, avec une valeur moyenne de 386,13 $\pm 27,57 \mu \mathrm{S} . \mathrm{cm}^{-1}$. Les eaux à forte conductivité sont celles du puits de Insafari (1039 $\left.\mu \mathrm{S} . \mathrm{cm}^{-1}\right)$, du puits de Tsernaoua (1083 $\left.\mu \mathrm{S} . \mathrm{cm}^{-1}\right)$ et du puits de Dangona $\left(1156 \mu \mathrm{S} . \mathrm{cm}^{-1}\right)$. La dureté des eaux souterraines du site varie entre $1,1^{\circ} \mathrm{F}$ et $63,5^{\circ} \mathrm{F}$, avec une moyenne de $15,68^{\circ} \mathrm{F}$. La dureté de l'eau est un indicateur du niveau de calcaire dans l'eau : elle correspond à sa teneur en calcium et en magnésium. Plus elle en contient, plus elle est « dure ». Cela révèle que les eaux sont aux limites douces et moyennement dure dans l'ensemble. Les eaux qualifiées de très dures sont les eaux du puits de Insafari $\left(42,1^{\circ} \mathrm{F}\right)$ du puits de Dangona $\left(63,5^{\circ} \mathrm{F}\right)$ et du puits de Tsernaoua $\left(53,5^{\circ} \mathrm{F}\right)$. Selon le guide OMS, boire de l'eau calcaire ou dure n'est pas contre-indiqué. L'eau calcaire participe à l'apport journalier en calcium dont l'organisme a besoin (solidification des os, fonctionnement des muscles, transmission de l'influx nerveux, processus de coagulation du sang). Les eaux des puits se caractérisent par la présence de matières en suspension responsables de la couleur qui varie entre 20 et $550 \mathrm{mg} / \mathrm{Pt}$ et de la turbidité qui varie respectivement entre 3 et 250 NTU. Les eaux sont riches en fer, avec des teneurs qui varient entre 0,05 et $3,20 \mathrm{mg} / \mathrm{L}$, pour une moyenne de $0,74 \mathrm{mg} / \mathrm{L}$. La plus forte valeur a été obtenue au niveau des eaux de puits de Gata Mata $(3,20 \mathrm{mg} / \mathrm{L})$. Le fer est indispensable pour le corps humain mais de très fortes concentrations affectent les propriétés organoleptiques de l'eau et tâchent aussi le linge. La valeur guide de l'O.M.S du Fer Total dans l'eau de boisson est de $0,3 \mathrm{mg} / \mathrm{L}$. Les points d'eau analysés ont une concentration en Fer $(0,74 \mathrm{mg} / \mathrm{L})$ supérieure à la valeur guide de l'OMS à l'exception de 8 localités soit $34,8 \%$ des eaux de la région. Un des éléments les plus importants dans l'eau de boisson et lié à la santé est le fluor. Celui-ci se présente dans l'eau sous forme d'ions fluorures ( $F-$ ). Les concentrations en $\mathrm{F}$ - inférieures à $0,5 \mathrm{mg} / \mathrm{L}$ dans l'eau de boisson peuvent favoriser la carie dentaire (Jordana et Batista, 2004). A de très fortes concentrations (au-dessus de 1,5 - $2 \mathrm{mg} / \mathrm{L}$ ), le fluor dans l'eau de boisson peut occasionner une fluorose dentaire ou osseuse (Brouwer et al., 1988; OMS, 2000). Dans les eaux analysées, les concentrations en $\mathrm{F}^{-}$se situaient entre $0,01 \mathrm{mg} / \mathrm{L}$ et $1,3 \mathrm{mg} / \mathrm{L}$. Les ions sulfate $\left(\mathrm{SO}_{4}{ }^{2-}\right)$ à des concentrations supérieures à la valeur guide dans l'eau de boisson peuvent provoquer des diarrhées chez l'être humain (CDC-EPA, 1999). L'analyse de nos données montre que les teneurs en sulfate peuvent être considérées admissibles à l'exception des puits de Tsernaoua (404,3 mg/L), de Dangona $(431,1 \mathrm{mg} / \mathrm{L})$ et de Insafari $(329,6 \mathrm{mg} / \mathrm{L})$ où elles dépassent la norme fixée par l'OMS qui est de $200 \mathrm{mg} / \mathrm{L}$. La contamination par les nitrates peut être considérée comme acceptable vis-à-vis de la norme $50 \mathrm{mg} / \mathrm{L}$. La présence des nitrites témoigne d'une contamination récente résultant de l'infiltration des eaux usées et d'un début de déficit du milieu en oxygène (Aghzar et al., 2001; Sinan, 2000). Les résultats de la contamination bactériologique par les coliformes fécaux montrent que la plupart des puits sont contaminés, ce qui montre le non potabilité de tous les puits.

Diagramme de PIPER: Le report de résultats des analyses des eaux des aquifères sur le diagramme triangulaire de Piper (Figure 2), met en évidence l'incidence du faciès lithologique sur la qualité des eaux et permet aussi d'estimer les pourcentages des éléments chimiques et leur classification. 


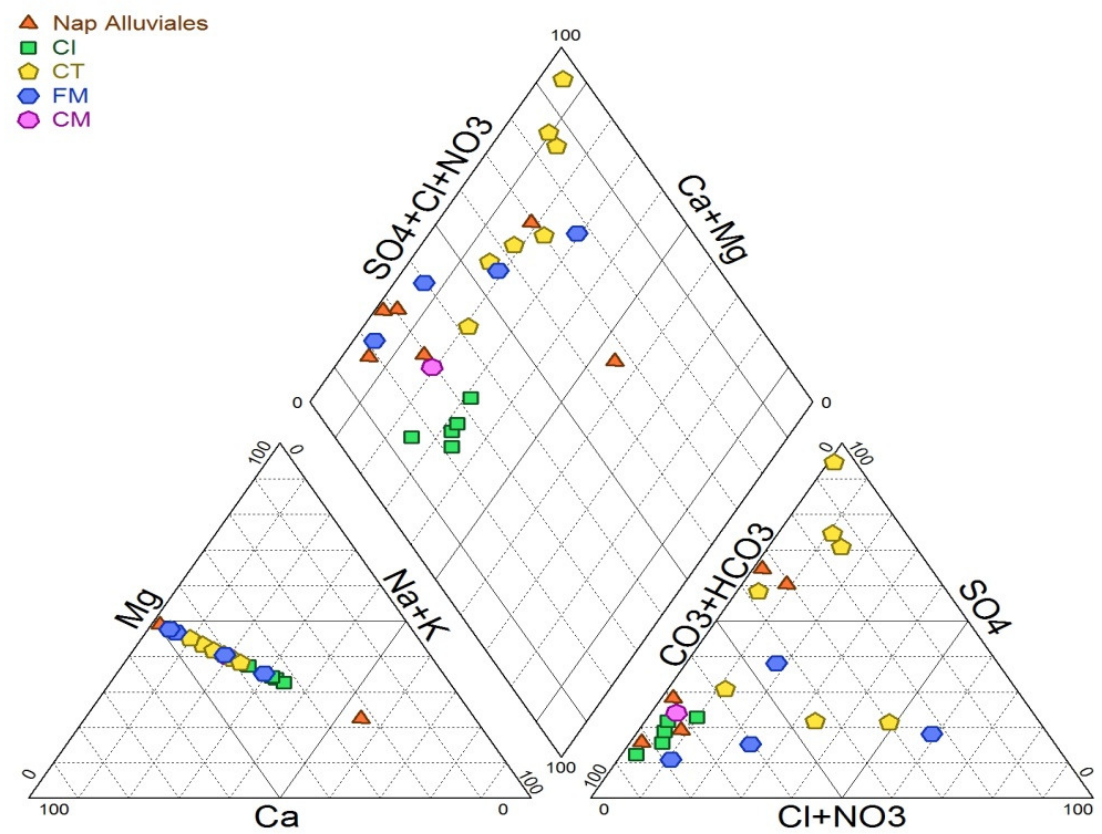

Figure 2 : Classification des eaux souterraines à partir du diagramme de Piper.

Ainsi le Diagramme triangulaire de Piper permet ainsi de distinguer: Les eaux bicarbonatées calciques et magnésiennes, les plus importantes $(60,87 \%)$ des eaux étudiées. Les eaux sulfatées calciques et magnésiennes $(21,74 \%)$ sont constituées des eaux du puits de Rididi, Bagga, Tsernaoua, Dangona, Doli. Les eaux chlorurées calciques et magnésiennes (13,04\%), sont constituées par les eaux du Badaguichiri, Kaoura Alassane, Tajae Nomade. Ensuite, viennent les eaux sulfatées sodiques (4,36\%) qui ne s'observent qu'au niveau du puits de Binguire. Dans la région, les eaux sont donc caractérisées par une prédominance des ions bicarbonates sur les ions sulfates et les chlorures. Le calcium constitue le cation le plus important, puis vient ensuite $\mathrm{Na}^{+}$.

Analyse en composantes principales Normées (ACPN) : L'étude statistique à partir de l'ACPN donne de nombreux résultats qui sont présentés dans les Tableaux 1, 2 et 3 . Dans le Tableau 1 sont consignés les valeurs propres, les variances exprimées pour chaque facteur et leurs cumuls. Le facteur $\mathrm{F} 1$, avec une variance exprimée de $38,90 \%$, est le plus important de tous, ensuite viennent les facteurs F2 et F3, avec respectivement $20,16 \%$ et $15,40 \%$ de la variance exprimée.

Tableau 1 : Valeurs propres et pourcentages exprimés pour les axes principaux.

\begin{tabular}{llll}
\hline & $\mathrm{F} 1$ & $\mathrm{~F} 2$ & $\mathrm{~F} 3$ \\
\hline Valeur propre & 5,056 & 2,621 & 2,002 \\
\% Total de la variance exprimée & 38,893 & 20,159 & 15,404 \\
Cumul de la variance exprimée (\%) & 38,893 & 59,052 & 74,456 \\
\hline
\end{tabular}

Ces trois facteurs traduisent l'essentiel des informations recherchées et permettent de représenter de manière significative le nuage de points car la somme de la variance exprimée par ces facteurs est de $74,46 \%$. La contribution des différentes variables dans la définition des principaux facteurs est donnée par le Tableau 2. Chaque facteur est défini par un certain nombre de variables essentielles dans la mise en évidence du mécanisme de minéralisation des eaux. Ce tableau montre que le facteur F1, le plus important est défini par la conductivité 
électrique $\mathrm{CE}(r=0,97)$, la dureté $(r=0,93)$, les ions sulfates $\mathrm{SO}_{4}{ }^{2-}(r=0,83)$, les ions fluorures $\mathrm{F}^{-}(r=0,735)$, les ions chlorures $\mathrm{Cl}-(r=0,71)$ et les ions sodium $\mathrm{Na}^{+}(r=0,71)$ et $\mathrm{NO}_{3}{ }^{-}(0,64)$ corrélés à un degré moindre.

Tableau 1. Corrélations entre les variables et les facteurs.

\begin{tabular}{llll}
\hline & F1 & F2 & F3 \\
CE & $\mathbf{0 , 9 6 9}$ & $-0,070$ & 0,142 \\
pH & $-0,097$ & 0,485 & $-0,505$ \\
Couleur & 0,109 & $\mathbf{0 , 7 1 4}$ & 0,304 \\
Turbidité & $\mathbf{0 , 0 9 7}$ & $\mathbf{0 , 8 0 8}$ & 0,492 \\
SO4 & $\mathbf{0 , 8 2 7}$ & $-0,289$ & 0,408 \\
NO2 & 0,527 & 0,556 & $-0,339$ \\
Fe++ & $-0,304$ & 0,392 & $\mathbf{0 , 6 3 2}$ \\
F- & $\mathbf{0 , 7 3 5}$ & $-0,311$ & 0,454 \\
NO3 & $\mathbf{0 , 6 4 4}$ & 0,091 & $-0,452$ \\
HCO3- & 0,435 & $\mathbf{0 , 6 7 7}$ & 0,032 \\
Cl- & $\mathbf{0 , 7 1 1}$ & 0,235 & $-0,509$ \\
Dureté & $\mathbf{0 , 9 3 1}$ & $-0,203$ & 0,168 \\
Na+ & $\mathbf{0 , 7 1 7}$ & $-0,109$ & $-0,119$ \\
\hline
\end{tabular}

Des liens significatifs existant entre les différents paramètres sont donnés par la matrice de corrélation (Tableau 3). Ces liens traduisent les différentes corrélations qui existent entre les variables étudiées. En se basant sur le coefficient de corrélation critique $r=0.64$ (Mangin, 1974), la conductivité électrique est fortement corrélée à la dureté $(r=0,97)$. II est à noter que La conductivité électrique (C.E) décrit les sels inorganiques présents en solution dans l'eau. Quant à la dureté totale de l'eau, elle est rattachée principalement à la quantité de calcium et de magnésium dans l'eau. Ainsi la CE présente d'une part une corrélation avec les ions $\mathrm{SO}_{4}{ }^{2-}(0,86)$ et d'autre part avec $\mathrm{F}^{-}(\mathrm{r}=0,78)$ et $\mathrm{Na}^{+}(0,68)$.

Tableau 3 : Matrice de corrélation :

\begin{tabular}{|c|c|c|c|c|c|c|c|c|c|c|c|c|c|}
\hline $\begin{array}{l}\text { Variables } \\
\mathrm{CE} \\
\mathrm{pH}\end{array}$ & $\begin{array}{l}\text { CE } \\
\mathbf{1} \\
-0,169\end{array}$ & $\mathrm{pH}$ & Couleur & Turbidité & $\mathrm{SO} 4^{2-}$ & $\mathrm{NO}^{-}$ & $\mathrm{Fe}^{2+}$ & $\mathrm{F}^{-}$ & $\mathrm{NO}^{-}$ & $\mathrm{HCO}^{-}$ & $\mathrm{Cl}^{-}$ & Dureté & $\mathrm{Na}^{+}$ \\
\hline $\begin{array}{l}\text { Couleur } \\
\text { Turbidité }\end{array}$ & $\begin{array}{l}0,054 \\
0,104\end{array}$ & $\begin{array}{l}0,238 \\
0,235\end{array}$ & $\begin{array}{l}1 \\
0,744\end{array}$ & 1 & & & & & & & & & \\
\hline $\begin{array}{l}\mathrm{SO}_{4}{ }^{2-} \\
\mathrm{NO}_{2}^{-} \\
\mathrm{Fe}^{2+} \\
\mathrm{F}^{-} \\
\mathrm{NO}_{3}{ }^{-} \\
\mathrm{HCO}_{3}- \\
\mathrm{Cl}^{-} \\
\text {Dureté } \\
\mathrm{Na}^{+}\end{array}$ & $\begin{array}{l}\mathbf{0 , 8 6 2} \\
0,416 \\
-0,250 \\
\mathbf{0 , 7 8 4} \\
0,481 \\
0,439 \\
0,585 \\
\mathbf{0 , 9 7 5} \\
\mathbf{0 , 6 8 4}\end{array}$ & $\begin{array}{l}-0,340 \\
0,138 \\
-0,335 \\
-0,307 \\
0,158 \\
0,292 \\
0,069 \\
-0,227 \\
-0,014\end{array}$ & $\begin{array}{l}0,081 \\
0,255 \\
0,272 \\
-0,036 \\
0,123 \\
0,307 \\
-0,024 \\
-0,027 \\
0,106\end{array}$ & $\begin{array}{l}0,068 \\
0,255 \\
0,521 \\
0,085 \\
-0,040 \\
0,553 \\
-0,029 \\
0,006 \\
-0,084\end{array}$ & $\begin{array}{l}\mathbf{1} \\
0,104 \\
-0,164 \\
\mathbf{0 , 8 8 7} \\
0,341 \\
0,117 \\
0,266 \\
\mathbf{0 , 8 9 2} \\
0,592\end{array}$ & $\begin{array}{l}\mathbf{1} \\
-0,029 \\
0,042 \\
0,488 \\
0,562 \\
\mathbf{0 , 8 1 6} \\
0,350 \\
0,147\end{array}$ & $\begin{array}{l}1 \\
-0,126 \\
-0,319 \\
0,136 \\
-0,274 \\
-0,303 \\
-0,328\end{array}$ & $\begin{array}{l}\mathbf{1} \\
0,264 \\
0,122 \\
0,180 \\
\mathbf{0 , 8 3 7} \\
0,369\end{array}$ & $\begin{array}{l}\mathbf{1} \\
0,089 \\
\mathbf{0 , 7 1 5} \\
0,428 \\
0,525\end{array}$ & $\begin{array}{l}1 \\
0,450 \\
0,310 \\
0.243\end{array}$ & $\begin{array}{l}1 \\
0,512 \\
0,458\end{array}$ & $\begin{array}{l}1 \\
0.571\end{array}$ & 1 \\
\hline
\end{tabular}

Les ions $\mathrm{SO}_{4}{ }^{2-}$ possèdent une bonne corrélation avec la dureté-( $(r=0,89)$. Ce qui montre que les ions $\mathrm{SO}_{4}{ }^{2-}$ proviendraient de formations gypseuses. Les ions $\mathrm{F}^{-}$ sont corrélés avec la dureté $(0,83)$. Le fluor dans l'eau provient principalement de la dissolution des minéraux naturels présents dans les roches et les sols avec lesquels l'eau réagit (Ravindra et Garg, 2007). La fluorine $\left(\mathrm{CaF}_{2}\right)$, la cryolithe $\left(\mathrm{Na}_{3} \mathrm{AlF}_{6}\right)$, la fluo apatite $\left(\mathrm{Ca} \mathrm{F}_{5}\left(\mathrm{PO}_{4}\right)\right.$ et les micas sont les principaux minéraux qui le contiennent. Les ions $\mathrm{NO}_{3}{ }^{-}$sont corrélés avec $\mathrm{Cl}^{-}$ $(0,71)$. Les ions nitrate $\left(\mathrm{NO}_{3}{ }^{-}\right)$sont présents dans la nature où ils font partie du cycle de l'azote. Ils 
représentent la forme la plus soluble de l'azote. Principalement utilisés comme engrais inorganiques pour la croissance des plantes et la synthèse de composés azotés organiques, les nitrates en excès peuvent se retrouver rapidement dans les eaux souterraines. Les déchets contenant de l'azote organique représentent aussi une source de nitrates obtenus à partir de différents processus biochimiques (ammonification et nitrification) (Aghzar et al., 2001). L'espace des variables du plan factoriel F1-F2 (Figure 3) montre que ce plan exprime $59,05 \%$ de la variance exprimée. Le facteur $\mathrm{F} 1$ (38,89\%) est déterminé par $\mathrm{CE}$, dureté, $\mathrm{SO}_{4}^{2-}, \mathrm{F}-\mathrm{Cl}^{-}, \mathrm{Na}^{+}, \mathrm{NO}_{3}$ - Les éléments qui définissent ce facteur proviennent d'une longue durée de mise en solution suite au contact eau-roche. Ces éléments proviennent de l'hydrolyse des minéraux présents dans les roches qui constituent le substratum rocheux des aquifères qui abritent les eaux de la région. En effet, l'hydrolyse étant un processus lent, le facteur F1 exprime donc le phénomène de minéralisation-temps de séjour. Le regroupement de la majorité des variables soutenues par la minéralisation, autour de cet axe montre l'influence de l'altérationhydrolyse dans la mise en solution des ions. Le facteur F2 qui explique $20,15 \%$ de l'inertie du nuage des points représentatifs des ouvrages est déterminé par la couleur ( $r=0,71)$, la turbidité $(r=0,80)$, et $\mathrm{HCO}_{3}-(\mathrm{r}=0,67)$. La turbidité de l'eau souterraine est généralement inorganique (limon, argile et composés chimiques naturels tels que le carbonate de calcium) et causée par des facteurs géologiques naturels (Hossein, 2004).

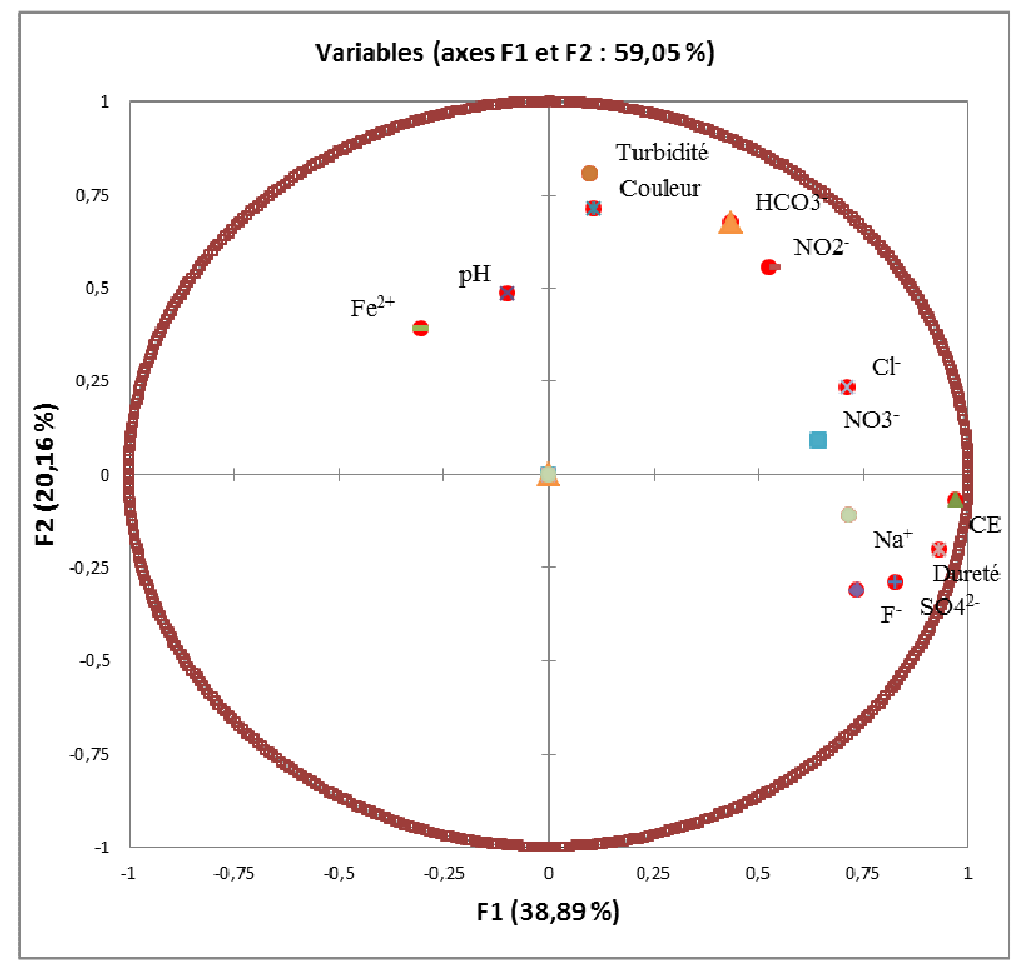

a) Espace des variables du plan factoriel F1-F2. 


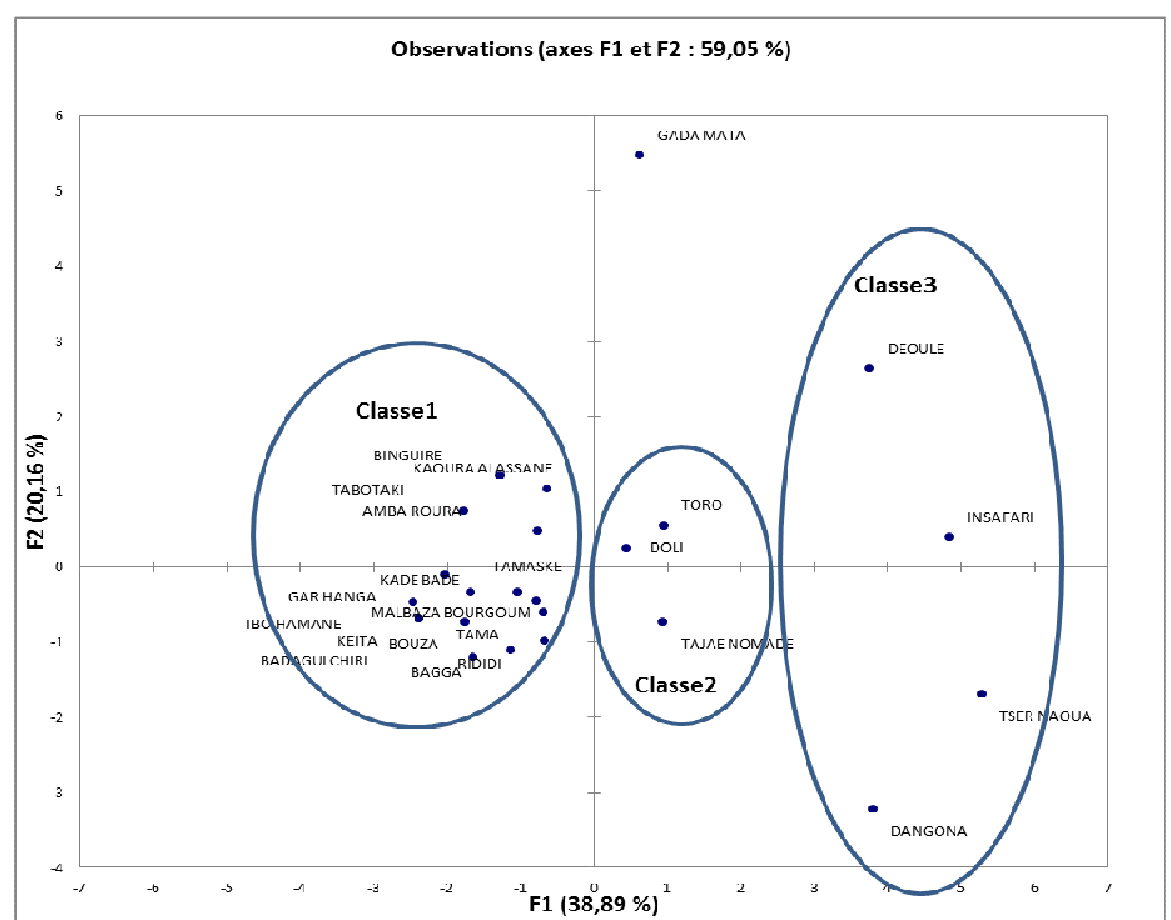

b) Espace des unités statistiques du plan factoriel F1-F2.

Figure 3 : Analyse en composantes principales des eaux dans le plan factoriel F1- F2.

Ce plan met en évidence les échanges superficiels qui se déroulent entre les eaux du site et les eaux de ruissellement issues des précipitations et du drainage des sols. Ces échanges mettent en évidence le phénomène de pluvio-léssivage des sols. Quant à l'axe $\mathrm{F} 3$, il n'est corrélé qu'aux ions ferreux à un degré moindre $(r=0,63)$ ce qui traduit un phénomène d'altération par hydrolyse des silicates. Le fer est issu du phénomène d'oxydo-réduction. Au vu du faible pourcentage exprimé par ce facteur nous pouvons dire que les mécanismes qui contrôlent l'évolution chimique des eaux de la région sont largement contenus dans les deux premiers facteurs. La représentation graphique dans l'espace des unités statistiques du plan factoriel F1-F2 (Figure 3) met en évidence trois principaux regroupements des points d'eau. La classe 1 contient la majorité des eaux de la zone d'étude. Ces points d'eau correspondent aux forages profonds et eaux de puits qui sont dans l'ensemble des eaux douces faiblement minéralisées. Les teneurs en bicarbonates de ces eaux sont faibles et présentent également des valeurs de conductivité électrique faibles. La classe 2 regroupe les eaux moyennement minéralisées de la région. Elle présente des caractéristiques d'eaux moyennement dures. La classe 3 regroupe des eaux de plus fortes conductivités électriques dont l'acquisition ionique est sous le contrôle de la minéralisation-temps de séjour : ce sont des eaux à caractéristique très dure de 3 puits Dangona $(1156 \mu \mathrm{S} / \mathrm{cm})$ Tsernaoua $(1083 \mu \mathrm{S} / \mathrm{cm})$ et Insafari $(1039 \mu \mathrm{S} / \mathrm{cm})$. Ces eaux sont fortement minéralisées mettant en évidence le phénomène de dissolution de la roche par les mécanismes d'hydrolyse. Elles sont aussi caractérisées par une présence de turbidité de teneurs supérieures à la Norme, des traces de fer, de nitrates et aussi des germes de contamination fécale. Le Dendrogramme (Figure 4) issu de la Classification Ascendante Hiérarchique (CAH) met en évidence deux principaux regroupements des variables. Le premier regroupement est constitué de $\mathrm{CE}$, dureté, $\mathrm{F}$-, $\mathrm{SO}_{4}{ }^{2-}$, $\mathrm{Na}^{+}, \mathrm{NO}_{3}{ }^{-}, \mathrm{NO}_{2}$ - et $\mathrm{Cl}$. Ce groupe rend compte de la minéralisation-temps de séjour ou du phénomène de l'hydrolyse des minéraux. II indique aussi une présence massive de composés azotés $\mathrm{NO}_{2}^{-}$et $\mathrm{NO}_{3}$ - donc une forte contribution des activités anthropiques dans la minéralisation des eaux souterraines. Le second groupe constitué de $\mathrm{HCO}_{3}^{-}, \mathrm{Fe}^{2+}, \mathrm{pH}$, couleur, et turbidité indique la contribution des activités anthropiques dans la minéralisation des eaux souterraines de la région. Ce groupe met également en évidence le phénomène de minéralisation gouverné par l'infiltration des eaux de pluie dans les aquifères. 


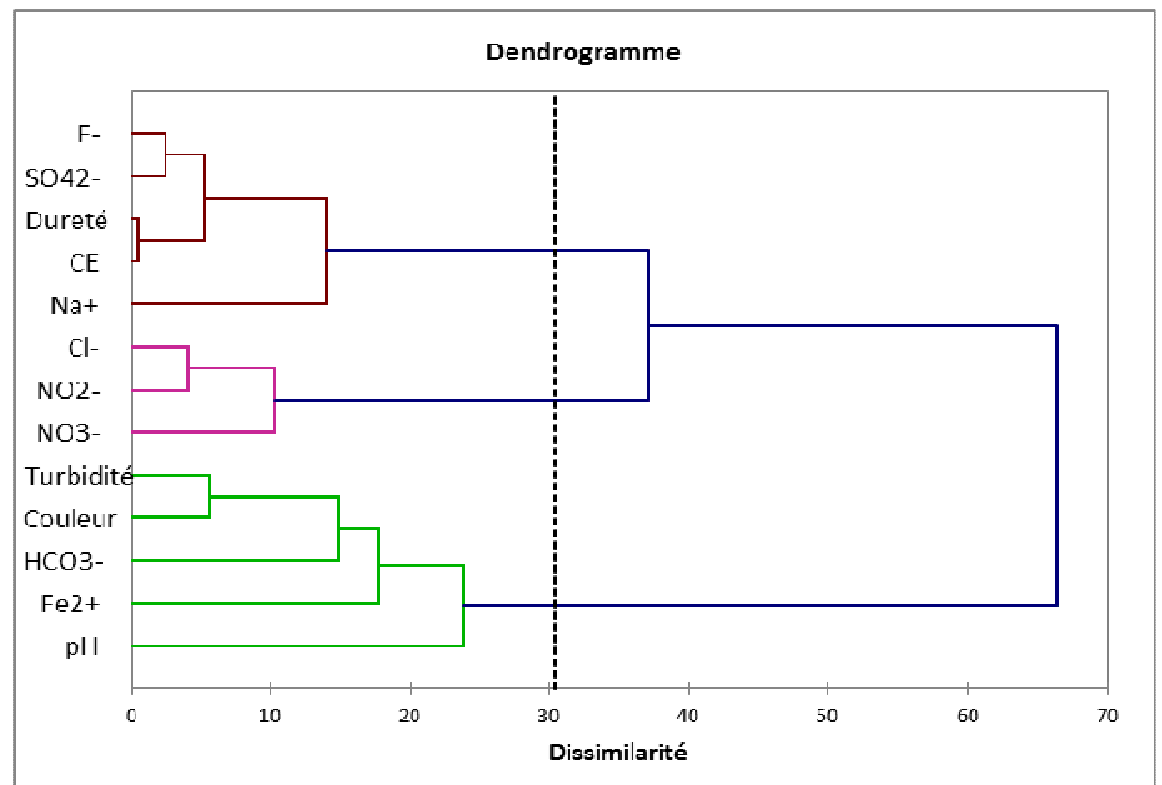

Figure 4 : Dendrogramme des eaux de la zone étudiée.

Analyses microbiologiques: Les résultats des analyses microbiologiques montrent la présence des bactéries de types coliformes fécaux (Escherichia coli) et streptocoques fécaux et Clostridium Perfringens, dans l'eau. Ces bactéries se retrouvent dans tous les puits étudiés. Les concentrations varient entre de 0 à 7200 UFC pour $100 \mathrm{ml}$ pour les coliformes fécaux; et de 0 à 140 UFC pour $100 \mathrm{ml}$ pour les streptocoques

\section{DISCUSSION}

L'étude hydro chimique a permis de mettre en évidence la prédominance de faciès bicarbonaté dans les eaux souterraines de la zone Tahoua. Dans cette région, les eaux souterraines se regroupent en quatre principaux hydro faciès: Les eaux bicarbonatées calciques et magnésiennes sont les plus importantes $(60,87 \%)$ des eaux étudiées. Les eaux sulfatées calciques et magnésiennes $(21,74 \%)$, les eaux chlorurées calciques et magnésiennes $(13,04 \%)$, et les eaux sulfatées sodiques $(4,36 \%)$.

La matrice de corrélation (Tableau 2), indique que les paramètres chimiques influençant la minéralisation des eaux de la zone sont le bicarbonate, la conductivité électrique, le Fluor, le sodium, la dureté (liée à la présence du calcium et du magnésium) et le chlorure. Ces ions sont issus de l'altération des roches et de l'hydrolyse des minéraux silicatés (Savané et al., 2001; Guler, 2004). Des ions comme $\mathrm{Cl}, \mathrm{SO}_{4}{ }^{2-} \mathrm{NO}_{2}$ - et $\mathrm{NO}_{3}{ }^{-}$ proviennent de la pluie et du lessivage des sols par celle-ci comme l'indique les résultats de l'analyse multi fécaux. Cela montre que les eaux de la zone d'étude sont soumises à une influence anthropique. En effet, la valeur indicative pour la qualité bactériologique proposée par l'Organisation Mondiale de la Santé (OMS) est de zéro coliforme pour $100 \mathrm{ml}$ d'eau or des teneurs supérieures à cette valeur ont été observées dans les eaux du site.

variée. En effet, l'Analyse en Composantes Principales a monté que deux autres phénomènes participent à la minéralisation des eaux de la zone d'étude. II s'agit du pluviolessivage des sols et de l'intervention des activités anthropiques dans la pollution des eaux des nappes superficielles. La présence des coliformes fécaux du type Escherichia coli dans les eaux de la zone d'étude rend compte de l'effet d'une pollution bactériologique récente à laquelle les eaux sont soumises. Dutka et al. (1990) associent l'abondance des coliformes fécaux dans une eau à une pollution bactérienne anthropique qui se produit lors des puisages par les populations. Les analyses physicochimiques montrent que pour une nappe donnée les différents points d'eau pris comme échantillon présentent souvent des caractéristiques très variables d'un point à un autre.

Les eaux des alluviales de fond de kori présentées par les eaux de Doli et Gada Mata se classent dans la classe 2. Ce sont des eaux carbonatées avec une 
minéralisation moyennement accentuée. Les eaux de Insafari sont deux fois plus carbonatées et deux fois plus minéralisées se trouvent dans la classe 3 alors que les eaux de Doli et Gada Mata ont des concentrations en sulfates très admissibles et, celles de Insafari dépassent de loin les normes OMS pour ce paramètre.

Les eaux des alluviales de la Maggia (Tama et Malbaza Bourgoum) très peu minéralisées, correspondent à celles de la classe 2 . Elles sont tout de même alcalines et carbonatées.

Les eaux des alluviales de bas fond présentées par un seul point d'eau (Binguire) contrairement aux autres alluviales, sont faiblement minéralisées, très faiblement carbonatées et de la classe 1.

Les aquifères du continental intercalaire de la région de Tahoua ont été échantillonnées au niveau des villages de Keita, Tamaské, Bouza, Ibohamane et Kadé badé. Ces points d'eau correspondent aux forages et appartiennent à la classe1 faiblement minéralisées. Elles présentent des teneurs en fer très importantes pouvant aller jusqu'à 10 fois la valeur maximale admissible. Ce sont des eaux très ferrugineuses avec une faible concentration en carbonate.

Le continental terminal a été échantillonné au niveau des puits cimentés. On peut classer les eaux de cet aquifère en deux catégories :

\section{CONCLUSION}

L'étude des caractéristiques hydrogéochimiques des ressources en eau de la zone de Tahoua a été réalisée à partir de la combinaison des méthodes hydro chimiques, hydrogéologiques et de l'analyse statistique multi variée. Cette étude met en évidence les différentes caractéristiques physico-chimiques et bactériologiques des eaux du site. Cette étude relève que les eaux souterraines sont à la limite entre les eaux douces et les eaux moyennement dures, avec une moyenne de $15,68^{\circ} \mathrm{F}$. Les eaux du site sont également riches en fer, avec une moyenne de $0,74 \mathrm{mg} / \mathrm{L}$ de teneurs. La classification des résultats des analyses chimiques obtenues à partir du diagramme triangulaire de Piper permet d'identifier quatre groupes d'eau. Les eaux bicarbonatées calciques et magnésiennes, les plus importantes $(60,87 \%)$ des eaux étudiées. Les eaux sulfatées calciques et magnésiennes $(21,74 \%)$, les eaux chlorurées calciques et magnésiennes $(13,04 \%)$, et les eaux sulfatées sodiques $(4,36 \%)$ qui sont les moins importantes. La représentation graphique dans l'espace factoriel des unités statistiques a mis en évidence trois (3) classes d'eaux. La classe 1 contient
La première catégorie regroupe les villages de Dangona et Tsernaoua. Ces villages sont caractérisés par des eaux très minéralisées (conductivité supérieure à $1000 \mu \mathrm{s} / \mathrm{cm}$ ), très carbonatées, très dures avec des concentrations en sulfates très élevées. Le fer se trouve à la limite de la norme. Elle appartient aux eaux de la classe 3 . La deuxième catégorie regroupe les villages de Rididi, Badaguichiri, Bagga et Kaoura Alhassan. Contrairement à la première catégorie, les eaux de cette catégorie sont faiblement minéralisées et faiblement carbonatées appartiennent à la classe 1.

Les formations marines de la région de Tahoua ont des compositions très variables d'un endroit à un autre. On note des minéralisations faibles à Tabotaki et Amba Roura (classe1) et des minéralisations élevées à Deoulé (classe3) en passant par des minéralisations moyennes à Tajae Nomade (classe2). Seules les eaux de Deoulé ont un caractère prononcé en carbonate. On note tout de même des concentrations en fer dépassant la limite admissible à Tabotaki. Deoulé et Tajaé Nomade présentent des eaux à concentrations en nitrates qui se trouvent à la limite des valeurs admissibles.

Le crétacé marin a été échantillonné au niveau d'un seul village à savoir le village de Toro. Ces eaux sont moyennement minéralisées et carbonatées.

la majorité des eaux de la zone d'étude. Ces points d'eau correspondent aux forages profonds et eaux de puits qui sont dans l'ensemble des eaux douces faiblement minéralisées. Les teneurs en bicarbonates de ces eaux sont faibles et présentent également des valeurs de conductivité électrique faibles. La classe 2 regroupe les eaux moyennement minéralisées de la région. Elle présente des caractéristiques d'eaux moyennement dures. La classe 3 regroupe des eaux de plus fortes conductivités électriques dont l'acquisition ionique est sous le contrôle de la minéralisation-temps de séjour. L'Analyse en Composantes Principales Normées (ACPN) et la Classification Hiérarchique Ascendante (CHA) indiquent que la minéralisation des eaux de la région est contrôlée par trois grands phénomènes. II s'agit de la minéralisation-temps de séjour ou l'hydrolyse pour les eaux souterraines (forages et puits), du pluviolessivage des sols pour les eaux de puits et de l'influence des activités humaines dans la production des déchets polluants. Par ailleurs, les eaux de la zone d'étude contiennent de forts taux de coliformes fécaux 
(Escherichia coli) et de streptocoques fécaux qui sont la conséquence d'une pollution d'origine humaine récente.

Ces travaux permettront de disposer d'une base de données pour un suivi de la qualité physico-chimique et

\section{REFERENCES}

Aghzar N, Bellouti H et Soudi B, 2001. Pollution nitrique des eaux souterraines au Tadla (Maroc). Rev. Sci. Eau, 15(2) 459-492.

Ahoussi K. E., Koffi Y. B., Kouassi A. M., Soro G., Biemi J. (2013). Étude hydrochimique et microbiologique des eaux de source de l'ouest montagneux de la Côte d'Ivoire : Cas du village de Mangouin-Yrongouin (Souspréfecture de Biankouman). Journal of Applied Biosciences; 63: 4703-4719.

Ahoussi K.E, Oga Y.M.S, Koffi Y.B, Kouassi A.M, Soro $\mathrm{N}$ et Biemi J. 2011. Caractérisation hydrogéochimique et microbiologique des ressources en eau du site d'un Centre d'Enfouissement Technique (CET) de Côte d'Ivoire : cas du CET de Kossihouen dans le District d'Abidjan (Côte d'Ivoire) ) . International Journal of Biological and Chemical Sciences, 5(5): 2114-2132.

Ahoussi M.A, Koffi E.K, Ake B. Y et Biemi J. 2012. Caractérisation hydrogéochimique des eaux des aquifères fissurés de la zone GuigloDuekoué (Ouest de la Côte d'lvoire). International Journal of Biological and Chemical Sciences. 6(1): 504-518.

Alayat H, Lamouroux C. 2007. Caractérisation physicochimique des eaux thermo-minérales des monts de la Cheffia (extrême Nord-Est algérien). La Presse thermale et climatique, 144: 191- 199.

Biémi J., 1992. Contribution à l'étude géologique, hydrogéologique et par télédétection des bassins versants Subsahéliens du socle précambrien d'Afrique de l'Ouest : Hydrostructurale, hydrochimie et isotopie des aquifères discontinus des sillons et aires granitiques de la Haute Marahoué (Côte d'lvoire). Thèse de Doctorat d'EtatÉtat es Sciences Naturelle, Université Nationale de Côte d'Ivoire, p. 480.

Boubakar Hassane A. 2010. Aquifères superficiels et profonds et pollution urbaine en Afrique: Cas de la communauté urbaine de Niamey bactérienne des eaux souterraines de la région de Tahoua, mais méritent d'être complétés par d'autres investigations notamment une étude des paramètres chimique de pollution, les métaux lourds ainsi que les pesticides.

(NIGER). Thèse de doctorat, Université Abdou Moumouni de Niamey, p. 249.

Brouwer I.D., DE Bruin A., Dirks O.A., Hautvast J, 1988. Unsuitability of WHO guidelines for fluoride concentration in drinking water in Senegal. Lancet, 1, 223-225.

CDC-EPA, 1999. Health effects from exposure to high levels of sulfate in drinking water study. EPA 815-R-99-001, January.

Dutka BJ, Palmateer GA, Janzen EM, Sakellaris M. 1990. The presence of bacteria virus in groundwater and treated drinking water. Environmental Pollution, 63: 293-298.

Eblin S.G., Sombo A.P., Soro G., Aka N., Kambiré O., Soro N. (2014) Hydrochimie des eaux de surface de la région d'Adiaké (sud-est côtier de la Côte d'lvoire). Journal of Applied Biosciences 75:6259- 6271.

Foster S.S.D, 1995. Groundwater for development - an overview of quality constraints». In H. Nash \& G.J.H. Mc Call (Eds.), Groundwater quality. 17 th Special Report, London United: Chapman and Hall, 1-3.

Greigert J., 1966. Description des formations crétacées et tertiaires du bassin des lullemmeden (Afrique Occidentale). Direction des mines et la Géologie de la République du Niger, publ. no 2. BRGM, Paris, 237 p.c. à $1 / / 000000$.

Guler C and Thyne G. D, 2004. Hydrologic and geologic factors controlling surface and groundwater chemistry in Indian Wells-Owens Valley area, southeastern California, USA". Journal of Hydrology, Vol. 285 177-198.

Hossien M.T, 2004. Hydrochemical evaluation of groundwater in the Blue Nile Basin, eastern Sudan, using conventional and multivariate techniques. Hydrogeology Journal, Vol.12 144-158.

Jain P, Sharma J.D, Sohu D, Sharma P, 2005. Chemical analysis of drinking water of villages of Sanganer Tehsil, Jaipur District. Int. J. Environ. Sci. Tech., Vol. 2, №4 373-379. 
Jordana S and Batista E, 2004. Natural groundwater quality and health. Geologica Acta, Vol.2, $\mathrm{N}^{\circ} 2$, 175-188.

Kouassi AM, Yao KA, Ahoussi KE, Seki CL, Yao NA, Kouassi Kl. 2010. Apports des méthodes statistiques et hydrochimiques à la caractérisation des eaux des aquifères fissurés de la région du N'zi-Comoé (CentreEst de la Côte d'Ivoire). International Journal of Biological and Chemical Sciences, 4(5): 1816-1838.

Lallahem S. 2002. Structure et modélisation hydrodynamique des eaux souterraines : application à l'aquifère crayeux de la bordure nord du bassin de paris. Thèse de Doctorat, Université de Lille, France, p.243.

Mangin A, 1974. Contribution à l'étude hydrodynamique des aquifères karstiques. Concepts méthodologiques adoptés. Systèmes karstiques étudiés. Ann. Spéléol. 29, 4, 495.601.

Maoudé K, 1988. Contribution à l'étude hydrogéologique du Tadiss de Tahoua. Institut de géologie Centre d'hydrogéologie Université de Neuchâtel, 220p.

Mor S, K. Ravindra, R.P. Dahiya, A. Chandra, 2006. Leachate characterization and assessment of groundwater pollution near municipal solid waste landfill site. Environ Monit Assess, $\mathrm{N}^{\circ} 118,435-456$ [224].
O.M.S, 2000. Directives de qualité pour l'eau de boisson. 2nd Ed., Vol 2, Critères d'hygiène et documentation à l'appui, 1050p.

OMS, 2008. Guidelines for Drinking-water Quality (Third edition, incorporating the first and second Addenda, Recommendations). OMS: Geneva, 1; 515.

Ravindra K., Garg V.K, 2007. Hydro-chemical survey of groundwater of Hisar City and assesment of defluoridation methods used in India». Environ Monit Assess, N¹32, 33-43.

Savané I, Soro N. 2001. Caractérisation chimique et isotopique des eaux souterraines du NordOuest de la Côte : recharge des aquifères discontinus de la région d'Odienné. Africa Géoscience Review, 8(4): 379-390.

Sinan M, 2000. Méthodologie d'identification, d'évaluation et de protection des ressources en eau des aquifères régionaux par la combinaison des SIG, de la géophysique et de la géostatistique. Application à l'aquifère du Haouz de Marrakech (Maroc) ", thèse d'état. Université Mohamed V de Rabat, Maroc.

Yermani M, Zouari K, Michelot JL, Mamou A, Moumni L. 2003. Approche géochimique du fonctionnement de la nappe profonde de Gafsa Nord (Tunisie centrale). Journal des Sciences Hydrologiques, 48(1): 95-108. 